\title{
How Do Non-core Companies in Emerging Industries Stand Out?
}

\section{——Take Douyin in the Field of Mobile Short Video as an Example}

\author{
Liu Ping ${ }^{1, a}$ \\ ${ }^{1}$ Business School of Qingdao Binhai University, West Coast New District, Qingdao, Shandong, China \\ aliupbh@163.com
}

\begin{abstract}
The development of emerging technologies and the promotion of emerging industries are important measures for my country to stand in the new era, implement new development concepts, build a new development pattern, achieve high-quality development, and improve international competitiveness. The rapid growth of non-core companies in emerging industries is conducive to expanding the scale of emerging industries and forming a virtuous competitive atmosphere within the industry. In order to promote the rapid growth of non-core companies in emerging industries, through a comparative analysis of the survival and development process of Douyin and Kuaishou, the innovation strategy of Douyin was discussed, and the secrets of emerging non-core companies in emerging industries that rely on innovation to stand out.
\end{abstract}

Keywords: emerging industries, core companies, non-core companies, Douyin, Kuaishou, short video

\section{新兴产业中非核心企业是如何脱颖而出的？ 以移动短视频领域的抖音为例}

\author{
刘苹 1 , a \\ '青岛滨海学院商学院, 西海岸新区, 青岛, 山东, 中国 \\ aliupbh@163.com \\ 摘要 \\ 发展新兴技术，促生新兴产业是中国立足新时代，贯彻新发展理念，构建新发展格局，实现高质量发展，提高 \\ 国际竞争力的重要举措。新兴产业中非核心企业快速成长, 脱颖而出有利于扩大新兴产业规模, 在产业内部形 \\ 成你追我赶的良性竞争氛围。为了促进新兴产业领域非核心企业快速成长, 通过对抖音生存发展过程中与快手 \\ 的对比分析，探讨了抖音的创新策略，揭示了新兴产业领域后发的非核心企业依靠创新脱颖而出的秘诀。
}

关键词：新兴产业；核心企业；非核心企业；抖音；快手；短视频

\section{1. 前言}

新兴产业主要源于新兴技术从知识形态向商业 产品及服务的逐步演化 ${ }^{[1]}$ 。在新兴产业中先驱企业往 往获得先发优势, 抢占制高点, 成为该领域的核心企 业, 但由于新兴产业本身在快速发展壮大的过程中,
核心企业的地位并不牢固，后发的非核心企业有着赶 超核心企业的机会，只要抓住机遇，就有可能在激烈 的竞争中脱颖而出, 如移动短视频领域的抖音赶超快 手的实例。移动短视频作为新的宣传和营销媒介, 不 仅被广大群众喜爱, 还获得了商家越来越多的青睐, 甚至成为了政府部门施政的窗口, 对社会影响力与日 俱增。以抖音为例分析在移动短视频新兴产业领域后 
发的非核心企业在激烈的竞争中脱颖而出, 赶超先发 的核心企业的发展策略, 有助促进新兴产业领域非核 心企业的成长。

\section{2. 相关的概念界定}

\section{1. 新兴技术与新兴产业}

在技术史上, 新兴技术是指建立在科学基础上的 革新, 它们可能创造一个新行业或改造某个老行业, 具有以下典型特征: 新近出现或正在发展, 能显著并 快速地形成新想法和新技术, 有较大的发展潜力, 将 对经济结构产生重大影响等 ${ }^{[2]}$ 。21 世纪, 新兴技术是 指最新发展的一系列最尖端的具有融合性特点的技 术, 如原来的语音、数据、视频技术互相交互影响, 创造更有效率的新技术 ${ }^{[3]}$ 。新兴技术显现了一国（地 区) 未来的发展契机和可能的变化趋势, 是影响发展 中国家（地区）实施经济赶超战略的重要因素 ${ }^{[4]}$ 。

新兴产业是指随着新的科研成果和新兴技术的 诞生并应用而出现的新的经济部门或行业 ${ }^{[5]}$ 。在创新 驱动下, 新兴产业主要源于新兴技术从知识形态向商 业产品及服务的逐步演化。新兴产业的培育和发展关 系到国计民生, 具有引领性、创新性和动态性。与传 统产业相比, 新兴产业更具有技术含量、高附加值和 资源集约性,代表了经济社会新的发展方向。新时代, 发展新兴技术, 促生新兴产业是中国实现产业结构转 型升级、提高国际竞争力的重要举措 ${ }^{[6]}$ 。随着 AI、AR、 $5 G$ 、区块链等新兴技术的进一步成熟和应用，短视频 行业带着这些新兴技术基因高歌猛进, 爆发式增长, 形成了新兴产业领域。

\section{2. 核心企业与非核心企业}

根据大量的文献研究和实地调研, 辽宁大学王伟 光教授带领的科研团队将同一产业内的企业大致分 为核心企业与非核心企业。

从产业创新网络视角: 网络中存在自组织的创新 分工情况, 核心企业在网络中扮演 “创新孵化器” 的 角色，而非核心企业则扮演 “实验应用场” 的角色。 核心企业对非核心企业具备控制力, 受到知识溢出在 双方之间的影响, 一方面会加强核心企业对非核心企 业的控制, 另一方面会出现 “核心企业控制力悖论”, 即非核心企业在知识溢出效应的影响下挣脱核心企 业的控制 ${ }^{[7]}$ 。

从创新能力综合评价的视角: 解决了对核心企业 与非核心企业类型划分定量困难的问题。利用设计调 查问卷, 通过对沈阳高技术产业 1379 家企业进行调 查, 从技术水平、知识吸收、研发模式、企业控制力 以及政策环境五个方面建立了企业创新能力评价体 系, 用定量方法划分企业类型, 将各指标均高于平均 值的企业定义为核心企业, 剩余企业定义为非核心企 $\underline{\underline{\mu}}^{[8]}$ 。
从创新价值链的视角: 根据企业控制创新价值链 的长度, 判断企业是核心企业还是非核心企业。这种 观点从多数学者所认可的掌握核心技术即为核心企 业的论断, 转向控制越多创新价值生成环节的企业则 越具备创新活动控制权, 即为核心企业, 掌握的创新 价值链较短则为非核心企业 ${ }^{[9]}$ 。

本文从市场地位、市场竞争力和市场份额角度界 定具有知识临近性的同一产业领域中企业类型。处于 核心市场地位、具有较强市场竞争力和最大市场份额 的企业为核心企业, 处于边缘的市场地位, 市场竞争 力较弱, 市场份额较小的企业为非核心企业。

\section{3. 移动短视频产业的兴起}

移动短视频是随着移动互联网领域新兴技术的 广泛应用而迅速发展起来的一个新兴产业领域。2011 年移动短视频产品首次在美国上线, 30 秒内的短视 频成了移动用户休闲、娱乐及交流的新形式。这些具 有较高创造性、趣味性、神秘感的移动短视频满足了 用户创作和表现欲望, 在用户之间形成了一个互相连 通的动态的网络世界 ${ }^{[10]}$ 。随后, 中国移动短视频也逐 步兴起, 形成了一个具有代表性的新兴产业领域, 其 发展大体经历了三个阶段。

第一阶段： 2012 至 2015 年, 初步兴起。2012 年, GIF 快手从工具应用转型为短视频社区平台。 2013 年, 新浪推出了秒拍, 滕讯推出了微视, GIF 快手改名为快手。2014 年, 美图推出美拍, 让用户 轻松制作和分享短视频。2015 年, 优酷推出美点, 一下科技推出小咖秀。这些应用推出后, 吸引了大量 的用户使用, 为移动短视频的爆发奠定了基础。

第二阶段：2016 至 2018 年，爆发式增长。2016 年 2 月, “快手” 爆炸式增长, 用户总数超过 3 亿, 成为移动短视频领域的核心企业; 2016 年 9 月，字 节跳动下属的北京微播视界科技有限公司推出抖音。 2017 年, 字节跳动又推出了西瓜视频, 参与移动短 视频领域的竞争。2018 年, 腾讯复活了微视。在各 大 App 应用激烈竞争角力的情况下抖音开始懪发, 在 App Store 上的排名快速超越快手，并突破了现象级 瓶颈, 成为了短视频领域具有强大实力的竞争者, 在 BAT 巨头纷纷布局的移动短视频领域, 逐渐改变了竞 争格局, 实现了对快手的快速赶超。

第三阶段： 2019 年至今，规范式发展。短视频 爆发式增长两三年后, 形成了几大派系创新竞争的局 面。其中包括腾讯系的快手、微视、火锅、闪咖等; 头条系的抖音、西瓜、火山、内涵段子等; 百度系的 好看视频、快手、全民小视频、榴梿等; 阿里系的土 豆、鹿刻、独客等; 新浪系的秒拍、酷燃视频、爱酷 小视频等。这些派系的短视频平台形成了三大梯队: 第一梯队为抖音与快手两家超强的头部企业; 第二梯 队为西瓜视频和火山小视频; 第三梯队为土豆视频、 波波视频、好看视频、微视、美拍、秒拍、快视频等。 2020 年受新冠肺炎疫情和国际关系纠纷影响，中国 
移动短视频企业在国内发展良好, 在国外的发展布局 受阻, 面临着新的机遇和挑战。

在移动短视频产业的兴起过程中, 可以清晰地看 出，抖音作为后发企业，相对于先发的核心企业快手 来说，一开始市场地位、市场竞争力和市场份额都次 于快手，是典型的非核心企业。通过快速地发展，抖 音仅仅用了两年左右的时间就实现了对快手的追赶 甚至超越, 成长为了短视频产业领域的新的核心企 业, 与快手并驾齐驱。抖音是如何脱颖而出的呢？现
将抖音和快手进行对比分析, 以窥其道。

\section{4. 抖音与快手的对比分析}

\section{1. 整体情况对比分析}

抖音在上线之初, 就与快手差别开来, 在定位、 理念、口号、产品形态、打开方式、算法逻辑及商业 模式等方面都有自己创新之处（如表 1)。其利用头 条数据资源和强大的技术支持快速地成长起来。

表 1 抖音与快手的对比

\begin{tabular}{|c|c|c|}
\hline 对比项 & 抖音 & 快手 \\
\hline 上线时间 & 2016 年 9 月 & 2011 年 3 月 \\
\hline 理念 & $\begin{array}{l}\text { 愉悦生活。热点运营、明星带流量、扶持 } \\
\text { 达人。 }\end{array}$ & 记录生活。轻运营、去大 V 化、坚持 UGC。 \\
\hline 口号 & 崇拜从这里开始一一记录美好生活。 & 记录世界，记录你。 \\
\hline 定位 & $\begin{array}{l}\text { 专注新生代年轻人的音乐短视频社区, 展 } \\
\text { 示的是年轻人的潮酷玩法, 是上层青年的 } \\
\text { KTV。聚焦头部内容创造者。 }\end{array}$ & $\begin{array}{l}\text { 记录与分享, 培育社区和社交, 展示年 } \\
\text { 轻人的活法, 是底层青年的生活圈。是 } \\
\text { 一个鼓励每个普通人展现自己的平台。 }\end{array}$ \\
\hline 卖点 & 带感音乐、炫酷技巧。 & 猎奇、趣味、搞怪。 \\
\hline 群众路线 & 一二线 $\rightarrow$ 三四线 $\rightarrow$ 小城镇、农村 & 小城镇、农村 $\rightarrow$ 三四线 $\rightarrow$ 二线 \\
\hline 产品形态 & $\begin{array}{l}\text { 抖音短视频搭配各种音乐类型、图像、文 } \\
\text { 字等多元信息, 内容具有很强的模板化效 } \\
\text { 果。用户个人才艺表演, 注重用户体验。 }\end{array}$ & $\begin{array}{l}\text { 照片+短视频+直播, 具有普惠性。内容 } \\
\text { 更加多样化, 注重分享。 }\end{array}$ \\
\hline $\begin{array}{l}\text { 内容生产 } \\
\text { 方式 }\end{array}$ & $\begin{array}{l}\text { UGC (用户生产内容) 、PGC （专业生产 } \\
\text { (专业用户生产内容) }\end{array}$ & 容）、OGC （职业生产内容） \\
\hline 打开方式 & $\begin{array}{l}\text { 全坚屏的节目播出, 下拉后, 自动播放。 } \\
\text { 抖音的一级入口分为首页、关注、消息、 } \\
\text { 我、拍摄视频和搜索。 }\end{array}$ & $\begin{array}{l}\text { 全坚屏的节目播出, 下拉后, 选择播放。 } \\
\text { 快手的一级入口分为首页、拍摄、菜单 } \\
\text { 按钮, 快手的消息、个人主页和搜索隐藏 } \\
\text { 在菜单按钮的下一级。 }\end{array}$ \\
\hline 算法逻辑 & $\begin{array}{l}\text { 高度中心化, 分发机制偏重观看者。关注 } \\
\text { 有特殊的设计, 突出作者头像和名字。 }\end{array}$ & $\begin{array}{l}\text { 强调普惠价值观, 强调好的内容得到更 } \\
\text { 多推荐, 不会向明星和红人进行流量倾 } \\
\text { 斜, 偏重记住者和观看者的平衡。 }\end{array}$ \\
\hline 娱乐效果 & $\begin{array}{l}\text { 通过视频特效、音乐剪辑, 使客户很容易 } \\
\text { 模仿热门的 “视频段子”。全屏高清、音 } \\
\text { 乐、特效滤镜、拍摄体验, 打造拥有 “美 } \\
\text { 好感” 的产品, 在传播属性上具有优势。 }\end{array}$ & $\begin{array}{l}\text { 取决于生活及行为本身的 “猎奇性” }, \\
\text { 对于绝大多数普通青年来说, 只能围观 } \\
\text { 而无法模仿。 }\end{array}$ \\
\hline 更新速度 & 持续快速升级, 创新玩法。 & 版本迭代速度较慢, 依靠用户开发玩法。 \\
\hline 商业模式 & $\begin{array}{l}\text { 网红打造出的头部市场, 即强运营、造话 } \\
\text { 题、聚焦头部网红、制造修行, 借助原有 } \\
\text { 平台倒流、圈粉。 }\end{array}$ & $\begin{array}{l}\text { 基于用户的长尾市场。所有分发全部基 } \\
\text { 于内容可关注度, 形成去中心化的 “老 } \\
\text { 铁” 经济。 }\end{array}$ \\
\hline
\end{tabular}

抖音于 2016 年 9 月上线, 经过半年左右时间的 酝酿, 于 2017 年 3 月第一次爆发, 2017 年 12 月, 实现第二次爆发, 此后, 抖音长期高居 iOS 排行榜前 几名。2018 年初, 抖音成为全球下载量最高的 App, 超过了 Facebook、YouTube 等应用。2018 年 6 月, 抖音在中国日活用户（DAU）突破了 1.5 亿，月活用 户（MAU）超过 3 亿，成为中国互联网短视频里面最 大的一款产品。2019 年，抖音的海外版 Tik Tok 在 全球下载量突破 7.38 亿次, 在苹果应用商店和谷歌 游戏应用商店中的下载量位居全球第二。到 2019 年 底抖音一共覆盖了全球超过 233 个国家和地区。2020 年 8 月, 抖音全球日活跃用户达到 5 亿, 发展非常迅
猛。新冠肺炎疫情期间抖音逆势而为, 成为了展现中 国文化、传递正能量和传播中国声音的平台。

\section{2. 用户特征对比分析}

\section{2. 1. 用户关注度变化趋势分析}

抖音晚于快手五年上线。上线之初其与快手在用 户关注度、使用量、市场份额、竞争能力等方面差距 明显。经过几轮的爆发增长, 差距逐渐缩小, 到了 2018 年第一季度过后, 抖音就已经追赶上了快手 (见 图 1), 在此之后, 抖音一度领先快手, 直到海外版 推广受阻, 发展势头才有所减弱。 


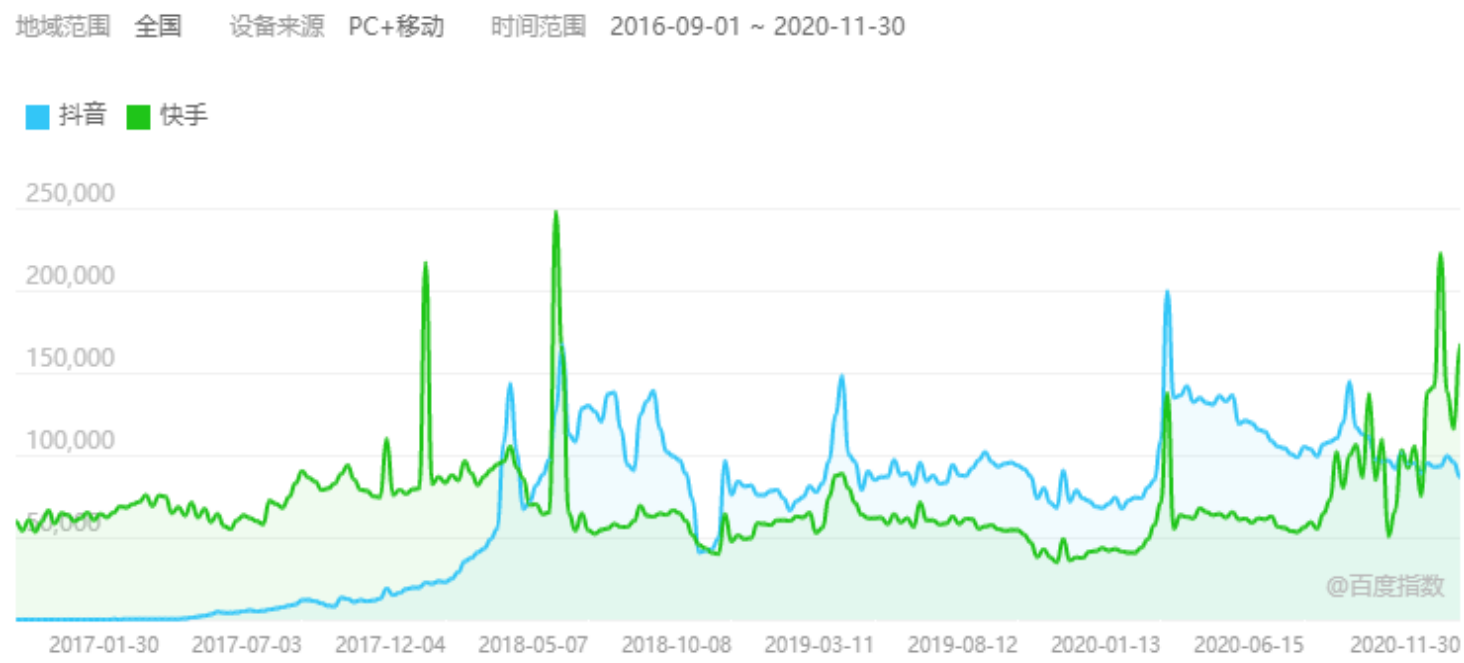

图 1 抖音和快手的用户关注度变化趋势图

数据来源：百度指数

\subsection{2. 用户地理分布分析}

由于抖音和快手的定位不同, 其用户在地里分布 上形成差异，抖音用户更多的集中在中国的华东、华

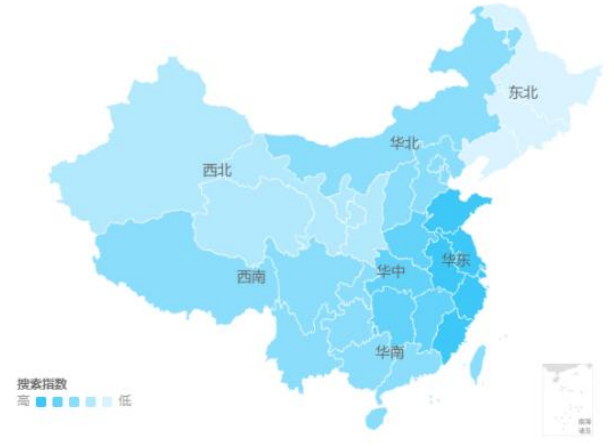

图 2 抖音和快手用户的地理分布图
中、华南、西南部分，而快手用户更多的集中在中国 的华北、西北、东北等地区（见图 2)，从而形成了 “南抖音, 北快手”的说法。

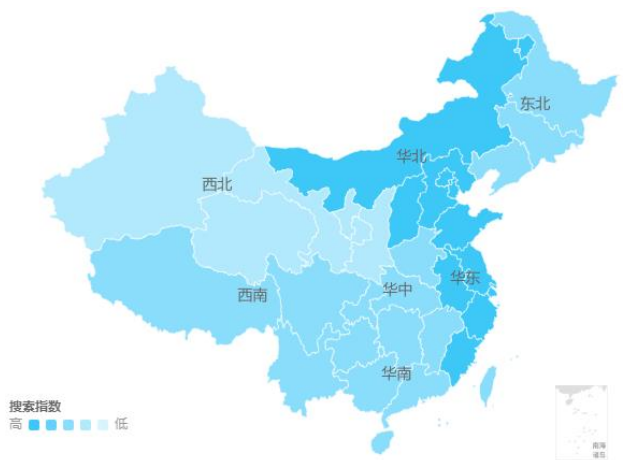

数据来源：百度指数

表 2 抖音和快手搜索用户居前十名的省市

\begin{tabular}{|c|c|c|c|c|}
\hline 排名 & \multicolumn{2}{|c|}{ 抖音 } & \multicolumn{2}{c|}{ 快手 } \\
\hline & 省份 & 城市 & 省份 & 城市 \\
\hline 1 & 广东 & 北京 & 河北 & 北京 \\
\hline 2 & 江苏 & 上海 & 山东 & 青岛 \\
\hline 3 & 河南 & 成都 & 广东 & 哈尔滨 \\
\hline 4 & 浙江 & 杭州 & 河南 & 石家庄 \\
\hline 5 & 山东 & 广州 & 江苏 & 上海 \\
\hline 6 & 四川 & 深圳 & 辽宁 & 保定 \\
\hline 7 & 河北 & 重庆 & 四川 & 广州 \\
\hline 8 & 湖北 & 郑州 & 浙江 & 成都 \\
\hline 9 & 北京 & 武汉 & 云南 & 郑州 \\
\hline 10 & 湖南 & 苏州 & 黑龙江 & 深圳 \\
\hline
\end{tabular}

\section{2. 3. 用户年龄和性别特征分析}

从用户年龄来看: 抖音和快手用户群体主要集中 于 20 岁到 40 岁, 尤其以二三十岁用户居多, 四十岁 以上群体抖音多于快手 (见表 3)。从用户性别来看: 抖音和快手都以男性用户为主，占比都超过 $60 \%$, 而 抖音的女性用户比快手女性用户多将近 10\%（见图 3)。

表 3 抖音和快手用户的年龄分布

\begin{tabular}{|c|c|c|c|c|c|}
\hline & $\leqslant 19$ 岁 & $\begin{array}{c}20-29 \\
\text { 岁 }\end{array}$ & $\begin{array}{c}30-39 \\
\text { 岁 }\end{array}$ & $\begin{array}{c}40-49 \\
\text { 岁 }\end{array}$ & $\geqslant 50$ 岁 \\
\hline $\begin{array}{c}\text { 抖 } \\
\text { 音 }\end{array}$ & $5.81 \%$ & $35.17 \%$ & $34.11 \%$ & $17.43 \%$ & $7.49 \%$ \\
\hline $\begin{array}{l}\text { 快 } \\
\text { 手 }\end{array}$ & $8.18 \%$ & $31.36 \%$ & $36.97 \%$ & $16.55 \%$ & $6.95 \%$ \\
\hline
\end{tabular}

数据来源：百度指数 


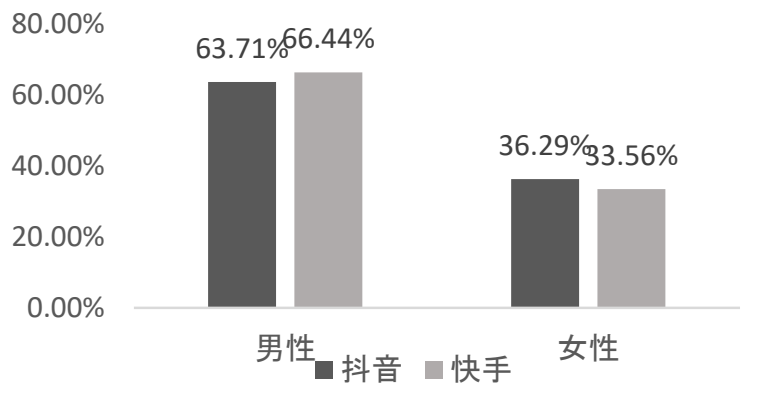

图 3 抖音和快手用户性别分布

\section{3. 发展的优劣势对比分析}

快手的出现和发展引领了内容消费形态从长视 频迁移到短视频时代, 让业内认识到十几秒短视频蕴 含的巨大想象力和商业潜力, 成为了该领域的先发的 领军企业。抖音的出现打破了这种局面, 以后发优势, 在激烈的竞争中, 异军突起, 对快手形成了有力的挑 战, 使得移动短视频领域形成了双雄割据的局面。抖 音和快手作为短视频领域的两家头部企业, 即有相同 之处, 更有明显的区别, 在竞争中, 各有优劣势。

\section{3. 1. 快手的优劣势}

快手具有先发优势, 打造出了一个长尾的内容市 场, 记录了生活中活生生的、新鲜的生活状态以及形 形色色的人和事, 更贴近百姓生活。在数据层面, 快 手比抖音先上线了很长时间, 积累了大量的短视频素 材。庞大的视频库将为基于图像的机器深度学习提供 基础, 为视频打出更细致的标签, 提升内容与用户匹 配的精准度 ${ }^{[11]}$ 。快手的劣势在于: 在短视频平台的竞 争中, 孤军奋战, 商业化落后与抖音, 内容存在大而 杂的问题。虽然有腾讯在背后的融资支持, 但难以阻 挡抖音等头条系短视频的崛起势头, 尤其在腾讯复活 微视后, 给予快手的融资支持将受到影响。

\section{3. 2. 抖音的优劣势}

抖音的优势: 在上线之初就依靠着今日头条积攒 的用户数据, 能够更好地预测用户喜好, 提高推荐适 配度, 延长用户的使用时间, 并且可以与今日头条系 共享客户资源。抖音在算法、运营、技术方面注重创 新, 也具有一定的优势, 这使得抖音得以快速跻身一 线短视频平台。同时, 抖音更看重的是用户体验, “优 质内容为王” 的逻辑更具有娱乐性和竞争性。在全球 化方面抖音的推进速度要比快手快。抖音的劣势在于 快速成长过程中对短视频内容的审核缺失。

\section{5. 抖音脱颖而出的创新策略}

后发的抖音利用了其具有后发优势, 通过观察快 手等前期短视频产品的市场反应, 更准确地把握消费 者痛点, 选择更加合理的差别化创新策略, 树立自身
独特的品牌, 快速地赶超快手, 创造了网络时代新的 发展奇迹, 这是抖音能够脱颖而出的秘籍。抖音快速 发展的过程就是不断创新的过程, 其开发、引领了新 时代年轻人的自我娱乐需求, 抖音的赶超创新策略主 要包括技术创新、内容创新、营销模式创新。

\section{1. 技术创新策略}

\section{1. 1. 优化中心化的算法, 培养 “抖红”}

最开始抖音仿效快手采用了去中心化的策略, 根 据用户喜欢和观看习惯推荐短视频, 以此保证平台上 的每个视频被看到的机会都是平等的, 用户不但是信 息的接受者更是信息的生产者, 不管用户是否具有知 名度, 只要制作的内容是优质的, 就会争取到更多的 曝光和播放机会, “收割” 大量关注度, 这种形式培 养出了最初的由大众篮选出来的 “网红” 和 “意见领 袖”，形成了一个个小 “中心”。在继续发展的过程 中，抖音的算法分发机制向这些中心人物倾斜，使他 们制作出来的优质内容更容易被用户看到, 成为一个 个 “抖红”。

\section{1. 2. 运用人工智能技术开发差别化的多样 功能, 增加客户棦性}

抖音依托今日头条的人脸识别、肢体识别等人工 智能技术开发出了包含短视频拍摄的特效、美颜、剪 辑、配乐等专业化的功能。这些功能在强大的技术创 新支持下，通过 “俊瓜式” 的操作呈现给用户，让每 一位普通用户通过创意、特长、精美拍摄而拥有成为 明星的机会, 在移动网络中获得真实的满足体验, 即 成了短视频的制作者、观赏者, 也是传播者 ${ }^{[12]}$ 。多样 化的功能给客户带来了个性化的服务, 使短视频产品 具有活力和吸引力, 能够充分调动用户的好奇心和兴 趣, 也吸引着越来越多的用户使用、探究和扩散。

\subsection{3. 设计了更富有感染力的打开和观看方 式，增强竞争力}

抖音认为全屏的表现模式一定是最优、最有冲击 力, 用户很容易就会被吸引。依靠后台强大的算法, 抖音给用户提供的是三选一, 有信心让用户在下滑两 三次以内选择到自己喜欢的内容。抖音利用大数据监 测和分析用户的性别、年龄分布、平均使用时间等, 根据用户的汶览习惯和感兴趣内容给用户推送相关 信息。这样的模式下, 相关视频内容就可以更加精确 的到达目标用户的使用终端, 同时也有利于更多的普 通用户获得更高的关注度和曝光量, 增加抖音在潜在 用户群体中的渗透率 ${ }^{[13]}$ 。用户在观看抖音短视频的时 候, 喜欢的内容可双击点赞, 提升了客户体验。另外, 在视频的整个页面只呈现出创作者的 ID、视频标题 和话题标签, 页面简洁且重点突出, 不会让用户感到 画面繁琐, 使得用户能够更容易地专心沉浸在视频观 看体验中。抖音依赖优质的观看效果和互动传播, 吸 
引着越来越多的用户 ${ }^{[14]}$ 。

\section{2. 内容创新策略}

\subsection{1. 满足用户体验性需求}

抖音依靠其强大的场景带入和沉浸式使用界面 给用户带来了移动终端下的沉浸式体验。其对用户使 用的场景要求低, 在家中、办公室、商场、餐厅、户 外等众多场所, 都可以作为抖音视频的录制背景和观 看背景。此外, 打开抖音 App, 视频内容就会自动播 放, 播放界面的内容是连续的视频, 从热门视频内容 到根据用户个人喜好所定制的内容持续不断的进行 播放。这会让用户在使用过程中沉浸在一种 “无意 识”、不间断的观看体验中, 用户被彻底拉进连续的 视频内容中，而忘记自己所处得时间和空间，从而 进入了一个沉浸的状态。

\subsection{2. 满足用户展示性需求}

抖音以年轻用户为主, 这些用户不仅对音乐、舞 蹈或视频剪辑有极高的热情和创造力, 而且群体归属 感和排外性都很强, 他们追求好玩的生活态度和有趣 的生活节奏, 具有个性、酷炫、新潮、狂拽、节奏感 强等特点。抖音的配乐以电音、舞曲为主, 视频分为 舞蹈派和创意派两个派别, 用户可以根据音乐短片和 自己的理解来进行二度创作，每个人的妆容、服装、 舞蹈和表情都有自己的特色和个性, 充分满足了受众 用户的展示性需求。

\subsection{3. 满足用户社交性需求}

抖音巧妙地创新了议程设置, 内容具有极高的继 承性、发展性和连贯性。抓住了内容的本质, 同时也 是抓住了用户的本质。抖音通过设置的点赞、关注、 评论等功能满足了用户的社交需求, 把志同道合的人 通过短视频连接在一起，使人们跨越了时空和年龄、 性别等界限, 在网络世界里广交朋友。

\section{3. 营销模式创新策略}

\section{3. 1. 紧跟潮流, 明星带流量}

抖音在推广过程中, 注重紧跟热点事件, 利用明 星效应推广自身。2017 年 3 月, 岳云鹏转发抖音视 频打响了明星带流量的第一声; 随后, 胡彦斌以新歌 为背景发起抖音挑战; 紧接着, 鹿晗携带新歌强势登 录抖音。 2018 年初, 抖音引入 10 位一线明星进行品 牌代言, 包括: Angelababy、吴亦凡、关晓栜、周冬 雨等, 带动了无数明星粉丝入驻抖音。

\section{3. 2. 制造热点，培育 “网红” 经济}

移动短视频给了每个人展示自我的机会, 创造了
大量的具有网络影响力的 “网红” 和 “意见领袖”。 这部分人作为网络群体中积极的信息交换者、内容生 产者, 成为了网络媒体环境中的焦点人物和意见导 向, 抖音积极培育从而在传播过程中实现 “再度中心 化” 成为新的意见领袖。这一部分人在短视频生产和 吸引用户层面被大众称为“网络红人”或者 KOL（Key Opinion Leader）。KOL 作为新媒体时代发展塑造 的关键意见领袖, 在平民化内容生产过程中起到了至 关重要的作用。抖音在发展阶段, 批量导入 KOL 和承 接头条的明星资源, 由明星和 KOL 去创造优质内容, 打造产品相关内容的娱乐 IP，增强用户黏性，从而 提高产品的生命力。

\subsection{3. 引爆话题, 借助多种媒介广泛宣传}

抖音于 2017 年 6 月在电视台上发布了第一支广 告, 此后抖音频频在各大电视台、网络及报纸上亮相、 曝光, 进行全方位广泛地宣传。抖音开展了一系列商 业合作，赞助《中国有嘻哈》《快乐大本营》等热门 综艺、与摩拜合作推出嘻哈主题单车等进一步增加品 牌懪光度 ${ }^{[15]}$; 线下举办抖音嘉年华等一系列活动, 邀 请网红、媒体参加, 形成线上、线下品牌互动。2019 年初抖音冠名湖南卫视春晚并成为央视春晚独家社 交媒体传播平台, 使抖音成为了家喻户晓的知名品 牌，成了老百姓茶余饭后的谈资。

\section{6 结论}

新兴产业领域企业之间的竞争更多地体现在创 新的内容、速度和质量方面。抖音通过创新快速地从 移动短视频领域脱颖而出, 但在发展的过程中也存在 着短视频内容良莠不齐、商业变现形式单一、红人孵 化机制不成熟等问题，抖音面对的强大竞争对手不仅 是快手，还有更加强大的腾讯、百度等巨头，随着 $A I 、 A R 、 5 G 、$ 区块链等新兴技术的进一步成熟和应用， 短视频产业的市场规模将成倍攀升, 如何在未来的竞 争中仍保持强大的竞争力, 建议抖音通过技术创新建 立更加严格的内容审核体系，进一步提高内容适配 度, 形成良好的社区生态, 进行模式创新更广泛地与 其他行业深度融合, 深化内容创新传播社会正能量, 成为新时代新风尚的塑造者。抖音作为非核心企业其 快速跃升的成功经验为新经济产业领域众多的非核 心企业的发展提供了参考和借鉴。非核心企业想要在 激烈的市场竞争中脱颖而出, 就必须要依靠融合性的 全方位创新, 与核心企业差别开来, 通过引领和满足 消费者个性化需求, 取得成功。

\section{项目基金}

本文为国家自然科学基金面上项目《创新价值链 视角下的非核心企业创新行为模式演化机理研究》 （71573113）的阶段性成果之一。 


\section{REFERENCES}

[1] [6] Chen F, Sui J G. Research on collaborative innovation and evolution of emerging industries: New energy vehicles as an example [J]. Scientific Research Management, 2015, 36 (01): 26-33.

[2] Dayg S,Paul J H,Schoemaker R E.Gunther.Wharton on Managing Emerging Technologies[M].New Jersey:Wiley,2000.

[3] Sheng Y C. Factor endowment, technical ability and catching up with latecomer technology [D]. Hunan University, 2008.

[4] Perez C, Soete L.Catching up in technology:entry barriers and windows of opportunity[M]/DOSI G,FREEMAN C,NELSON R,et al.Technical change and economic theory.London and New York:Pinter Publishers, 1988: 458-479.

[5] Lu X W. A Dictionary of Management: Shanghai Lexicographical Publishing House, 2013.

[7] Wang W G, Feng R K, Yin Bo. Can core corporate control in the industrial innovation network promote knowledge spillover? [J]. Management World, 2015 (06): 99-109.

[8] You L, Wang W G. Evaluation of non-core enterprises' technological innovation capability in the innovation network $[\mathrm{J}]$. Exploration of Economic Issues, 2017 (07): 54-63.

[9] Wang W G, Zhang Z Y, Hou Junli. Innovation value chain and its structure: a theoretical framework [J]. Science and Technology Progress and Policy, 2019, 36 (01): 36-43.

[10] Harris, Ryan.Instagram vs.Vine: Cut Through the Hype and Make Short Form Video Workfor You[J].KISSmetrics,2013,06:20.

[11] Wang X Q. Fast and steady movement [J]. Chinese Entrepreneurs, 2018 (15): 48-52.

[12] Fu K D Perspectives on the innovative strategies of short video social applications from the Douyin App [J]. New Media Research, 2018, 4 (09): 37-38.

[13] Li H Y. Research on the spread of Douyin App [J]. China Press, 2018 (02): 45-46.

[14] Ding Y. Douyin: the "dark horse" from "trembling" [J]. Shanghai Information Technology, 2018 (02): 72-75.

[15] Chen Fan. Research on Douyin Business Model Innovation[D]. Beijing Jiaotong University, 2020. 